\title{
Functional isotope imaging evaluation of terutroban efficiency in a pro- inflammatory rat model of subarachnoid haemorrhage (SAH)
}

Tonon D.', Triglia T.', Guarrigue P.', Bruder N.', Martin J.C. ${ }^{3}$, Alessi M.C. ${ }^{4}$, Velly L.'

'APHM, CHU Timone, Dept of Anaesthesiology \& Intensive Care, Marseille, France, ${ }^{2}$ Aix Marseille University, Vascular Research Center of Marseille INSERM UMRS 1076, CERIMED, Mars eille, France, ${ }^{3}$ CHU TIMONE Aix Marseille University, Laboratoire NORT INSERM UI 062 INRA UI 260, Mars eille, France, ${ }^{4}$ Aix Marseille University, Laboratoire NORT INSERM UI062 INRA UI 260, Marseille, France

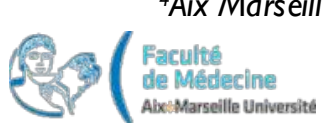

\section{BACKGROUND}

- Delayed cerebral ischaemia (DCI) is the first cause of morbidity after subarachnoid haemorrhage (SAH) [I].

- F2isoprostanes and eicosanoids were found in the cerebrospinal fluid (CSF) of patients with DCI. These potent vasoconstrictors induce platelet aggregation and mediate inflammation[2] by a thromboxaneprostaglandine (TP) receptor binding.

- The aim ofour study was first to estimate the occurrence of DCI in a proinflammatory state using an high omega 6 polyunsaturated fatty acid (w6) diet and secondly to evaluate the efficiency of terutroban (TER) a TP receptor inhibitor.

\section{MATERIALS AND METHODS}

- Ninety wistar rats $(400 \mathrm{~g})$ were randomly assigned to one of 5 groups: a double $250 \mu \mathrm{L}$ intracistemal injection of autologous arterial blood (SAH groups) or artificial CSF (CSF group) was performed [3].

- To induce a proinflammatory state animals were fat with w6 during 6 weeks before SAH procedure (SAH_w6/SAH_w6+TER). TER was administered $(30 \mathrm{mg} / \mathrm{kg} /$ day) during 5 days following $\mathrm{SAH}$ (SAH+TER/SAH_w6+TER groups).

- Evaluation of uptakes of 3 [99mTc] radiolabeled agents was achieved using microSPECT/CT imaging: HMPAO at D5 for cerebral perfusion quantification; DTPA at D3 for blood brain barrier (BBB) integrity study; and AnnexinV at D4 for apoptotic activity study. ANOVA followed by Student's t test.

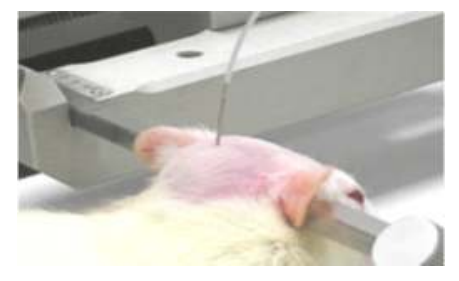

[Percutaneous puncture of the cisterna magna between the occiput and the atlas with the head held in a stereotaxic frame]

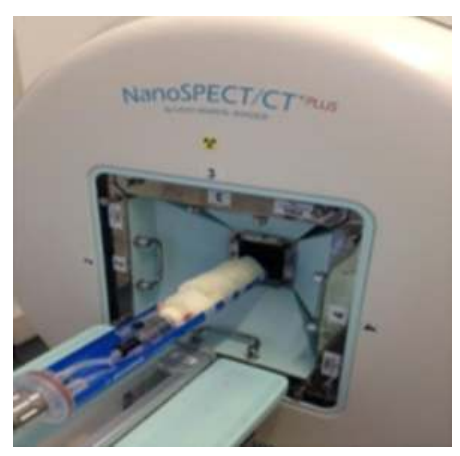

[microSPECT/CT imaging]

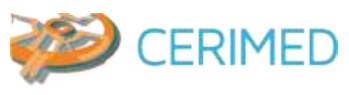

\section{RESULTS AND DISCUSSION}

- HMPAO uptake analysis showed a significant decrease in the SAH group (figure).

- DTPA and AnnexinV uptake were al so significantly increased in the SAH group compare to the CSF group. Proinflammatory state before SAH dramatically decreased HMPAO uptake (figure); increased DTPA $(0.37 \pm 0.04$ ws. $0.43 \pm 0.01 \mathrm{Mbeq} / \mathrm{mm} 3 ; P<0.05)$ and Annex in V $(0.39 \pm 0.03$ vs. $0.48 \pm 0.03 \mathrm{Mbeq} / \mathrm{mm} 3 ; P<0.05)$.

- TER significantly counteracted the decrease in HMPAO uptake (figure) and the increase in DTPA uptake $(P<0.05)$ and in AnnexinV uptake $(P<0.001)$ induced by SAH.

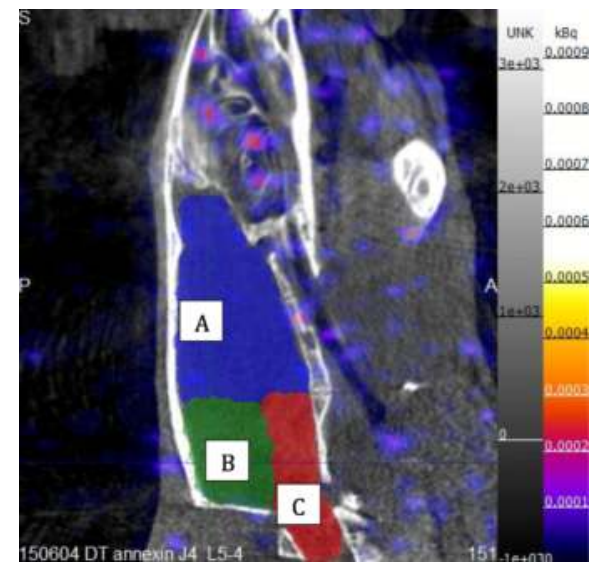

[ROIs of uptake analysis: A, Cerebral hemispheres; B, Cerebellum; C, Brainstem]

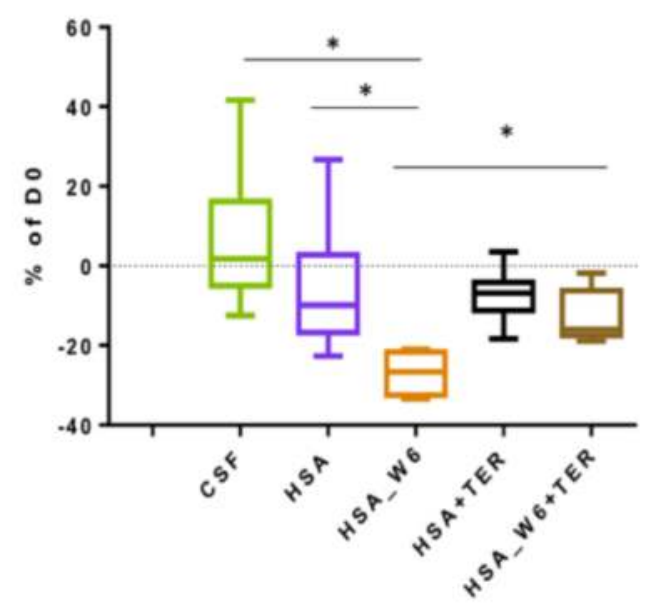

[Brainstem 99mTcHMPAO uptake at D5 expressed in \% of D0]

\section{CONCLUSION}

- For the first time, a proinflammatory SAH rat model of DCI has been described. microSPECT study shows that a proinflammatory diet dramatically increases apoptosis and DCI.

- TER improved hypoperfusion, BBB disruption and apoptosis. TP receptor antagonists could be promising treatments after SAH. 